\title{
Mammogram Images Enhancement using Adaptive Morphological Bilateral Filter
}

\author{
Roa I. Suliman \\ M.Sc Holder, Department of \\ Engineering \\ Faculty of Biomedical Engineering, \\ Sudan University of Sciences and \\ Technology \\ Khartoum, Sudan
}

\author{
Zeinab A. Mustafa, PhD \\ Assistance Professor, Biomedical \\ Engineering Department \\ College of Engineering, Sudan \\ University of Sciences and \\ Technology \\ Khartoum, Sudan
}

\author{
Banazier A. Ibraheem \\ Assistance Professor \\ Biomedical Engineering \\ Department \\ College of Engineering, Sudan \\ University of Sciences and \\ Technology \\ Khartoum, Sudan
}

\begin{abstract}
Noise, poor image contrast, in homogeneity, weak boundaries and special marks existing in the mammogram images makes diagnosis procedure extremely difficult, so there are needs for a way to denoise those images while preserving their important features. The Adaptive bilateral filter sharpens an image by increasing the slope of the edges without producing overshoot or undershoot. Morphological operations such as dilation, erosion, opening and closing with appropriate structure element size are offering a quality Sharpening enhancement. The performance of the filter was improved by including the mathematical morphology operations along with adaptive bilateral filter process. The parameters of the Adaptive bilateral filter are optimized with an iterative algorithm. The proposed method was applied for mammogram images. The performance analysis of the filter with respective design parameters and metrics are compared with existed algorithm the results were judged by three metrics; mean square error (MSE), structure similarity index (SSIM) and peak signal to noise ratio (PSNR).
\end{abstract}

\section{General Terms}

Image Enhancement, Noise filtration, Optimization.

\section{Keywords}

Adaptive bilateral filter, mammogram images, mathematical morphology, morphological opening, morphological closing, mean square error (MSE), peak signal to noise ratio (PSNR), structure similarity index (SSIM),

\section{INTRODUCTION}

Mammography is a low dose $\mathrm{x}$-ray procedure for the visualization of internal structure of breast. Mammography has been proven the most reliable method and it is the key screening tool for the early detection of breast cancer. Mammography is highly accurate, but like most medical tests, it is not perfect. On average, mammography will detect about $80-90 \%$ of the breast cancers in women without symptoms. [1]

Mammography is used for the early detection of masses in the breast and thereby reducing the death rate. There are different types of mammography like digital mammography, computer aided detection and breast tomosynthesis. Even though there are so many methods to diagnosing the breast cancer, it remains difficult to interpret some cases. Since the mammogram images are noisy and low contrast the radiologists may confused to diagnoses the cancer. In the present, the miss diagnosis rates of the radiologists about 10$30 \%$.[1]

The main problem of mammogram is that like other medical data it is also affected with noise during the acquisition of the mammogram images. So it is a challengeable task to denoise the mammogram images while preserving the important features of the image. The main noises affecting the mammogram images are salt and pepper, guassian, speckle and poisson noise. In previous days, noises in the mammogram images are denoised by the linear methods like mean and weiner filters. But the main problem of linear filtering is that it produces blurring effect and incomplete noise filtration. To overcome this limitation nonlinear filtering techniques were proposed.[2]

Bilateral filtering smoothes images while preserving edges, by means of a nonlinear combination of nearby image values. The method is non-iterative, local, and simple. It combines gray levels or colors based on both their geometric closeness and their photometric similarity, and prefers near values to distant values in both domain and range. $(\mathrm{ABF})$ is shown better performance than the ordinary form of the bilateral filters. This filter is modified with two important changes. One, an offset value is included along with range filter. Next both width and range of the adaptive filters are adaptive with respective output value. The bilateral filter proposed by Tomasi and Manduchi in 1998 is a nonlinear filter that smoothes the noise while preserving edge structures [3].

Methods of mathematical morphology act based on the structural properties of objects. These methods use mathematical principles and relationships between categories to extract the components of an image, which are useful in describing the shape of zones. Morphological operators are nonlinear, and two sets of data are their input. The first set contains the original image and the second one describes the structural element (mask). The original image is binary or in gray level and the mask is a matrix containing zero and one values.

It is after applying the final image to the morphological operators that a new value for each pixel is obtained through sliding the mask on the original image. Value 1 in each mask indicates effectiveness and value 0 indicates ineffectiveness in the final image. Different formats can be selected to form a mask.[4]

Morphological transform operation is carried out on medical images to enhance the contrast and quality while Adaptive bilateral filter removes noise from the images and gives 
significantly sharper image and by combining them optimum image enhancement can be achieve.

\subsection{Mathematical morphology (MM)}

Morphological operations used in digital image processing are a way of extracting image components that can be used to express details about a regions shape, its boundaries and its area. The value of each pixel in the output image is based on a comparison of the corresponding pixel in the input image with its neighbors.In general morphological operations are applied to binary images but there are extensions to the gray-level images.

Dilation and erosion are the two elementary operations in morphological processing; Erosion shrinks objects by eroding the boundaries. Dilation and erosion operations are performed by sliding a structuring element on the image. These two operators are inverse of each other [5]. An aggregation of these two represents the rest of the operations.

Dilation: (f B) $(x)=\sup \{f(x-y), y \in B\}$

Erosion: (f $B)(x)=\inf \{f(x+y), y \in B\}$

The processes of morphological dilation and erosion can be combined together in different ways to make interesting changes in images. Morphological opening and closing are two such operations that are defined by specific combinations of dilation and erosion.

Opening: $(f \circ B)(x)=((f \Theta B) \oplus B)(x)$

Closing: $(f \cdot B)(x)=((f \oplus B) \Theta B)(x)$

Morphological opening removes regions of an object that are smaller than the structuring element, smooth the edges of the object, and disrupts narrow connections. Similarly, morphological closing adds smoothness to image contours; however, it generally fuses two large regions separated by narrow breaks.

\subsection{Adaptive bilateral filtering}

The bilateral filter [6] is a nonlinear filter that does spatial averaging without smoothing edges. It has shown to be an effective image denoising technique. It also can be applied to the blocking artifacts reduction. An important issue with the application of the bilateral filter is the selection of the filter parameters, which affect the results significantly. Bilateral filter [6] is firstly presented by Tomasi and Manduchi in 1998. The ABF maintains the actual form of bilateral filter [7], in addition two significant modifications is included. The range filters is included an offset (w) function and width is introduced in domain filters. These gives the bilateral filter is spatially adaptive. The shift-variant filtering operation of ABF and its impulse response are shown in equations below.

$r\left(m_{0}, n_{0}\right)=$

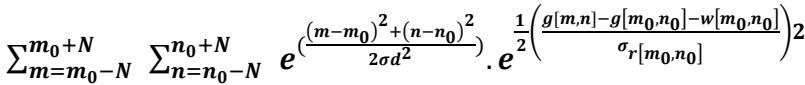

$h\left[m, n ; m_{0}, n_{0}\right]=$

$I\left(\Omega_{m_{0}, n_{0}}\right) r_{m_{0}, n_{0}}^{-1} e^{\left(\frac{\left(m-m_{0}\right)^{2}+\left(n-n_{0}\right)^{2}}{2 \sigma d^{2}}\right)} \cdot e^{\frac{1}{2}\left(\frac{g[m, n]-g\left[m_{0}, n_{0}\right]-w\left[m_{0}, n_{0}\right]}{\sigma_{r}\left[m_{0}, n_{0}\right]}\right) 2}$

Where $\left(\mathrm{m}_{0}, \mathrm{n}_{0}\right)$ is the center pixel of the window $\Omega_{\mathrm{m}_{0}, \mathrm{n}_{0}} \cdot \sigma_{\mathrm{d}}$ and $\sigma_{\mathrm{r}}$ are the standard deviations of the domain and range Gaussian filters, respectively. $\mathrm{r}_{\mathrm{m}_{0}, \mathrm{n}_{0}}$ is a normalization factor that assures that the filter preserves average gray value in constant areas of the image.

The main motivation to curry out this research was to is to improve the performance of the $\mathrm{ABF}$ by including mathematical morphology to enhance the mammogram image quality and filtering out nosise.

\section{METHODOLOGY}

Gray level mammogram images are considered for the proposed system. The approach is to extracting the image boundary using morphological operators then remove the noise through adaptive bilateral filter. The proposed method has the following steps:

First medical images are loaded into MATLAB environment and convert the gray scale values into Double precision format and normalize them in the interval $[0,1]$.Then produce a degraded image by adding Gaussian noise $\left(\sigma_{\mathrm{n}=0.05}\right)$ before the filtering process. There after selection of a proper size mask a series of mathematical morphological transforms like opening by reconstruction and closing by reconstruction are used to mark the valley and peak intensities of the gray scale values and improve the contrast.Then the bilateral filter parameters like half-width and domain range of filter might be set in terms of a standard deviation values an automatic model parameters selector approach was utilized which works by stacking all possible filter parameter values (standard deviation of the range filter, standard deviation of the domain filter, window size and offset) and then run the algorithm on each of them independently, comparing the results with the original image on each trail and then staking all PSNR values in a single column vector and picking the value that maximize the PSNR, and then pick its correspondent parameters. Then apply the adaptive bilateral filtering. Now calculate the performance metrics of the filter like the Mean Squared Error, PSNR and SSIM.

\section{RESULTS AND DISCUSSIONS}

All medical images are represented as gray scale images. Every pixel in gray scale images arise relative to the intensity or gray value. The main challenge in medical image analysis is to preserve the regions with edges and important features while remove the existing robust noise in the images.
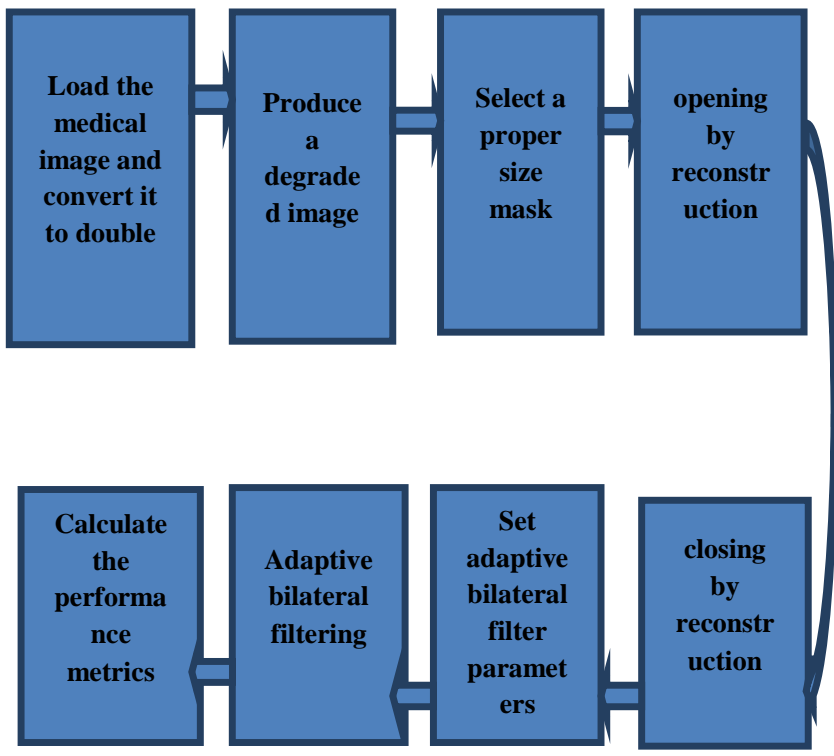

Fig.1 The Block diagram of the Proposed Method 
Experiments are performed using both $\mathrm{ABF}$ and $\mathrm{ABF}$ in combination with mathematical morphology operations and the results are shown.

These images are also evaluated by number of structure elements. The dimensions of the structure elements chosen are $3 \times 3,5 \times 5,7 \times 7$ etc. (a)

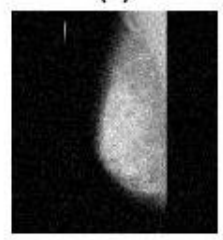

(b)

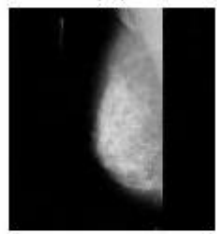

(c)

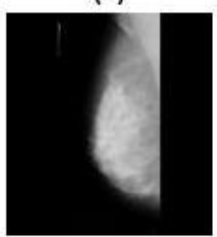

Fig.2 mammogram 1: G NORM :( a) Input image (b) ABF output (C) ABF with MM (a)

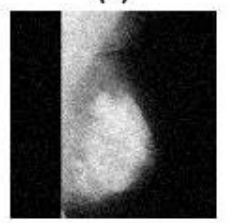

(b)

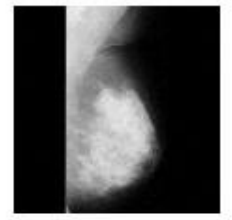

(c)

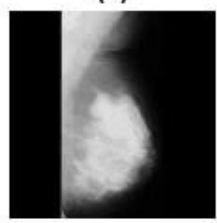

Fig.3 mammogram 2 D CIRC B: (a) Input image (b) ABF output (C) ABF with MM (a)

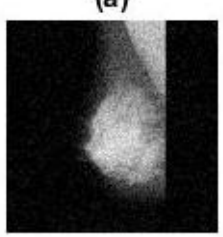

(b)

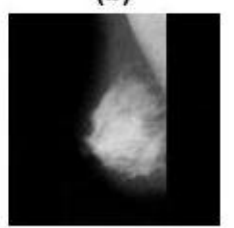

(c)

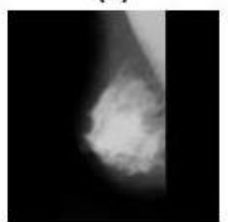

Fig.4 mammogram 3 G CIRC B: (a) Input image (b) ABF output (C) ABF with MM (a)

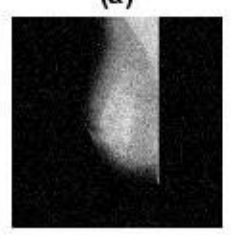

(b)

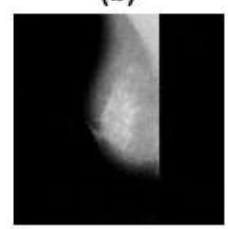

(c)

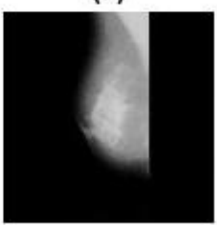

Fig.5 mammogram 4: G NORM : (a) Input image (b) ABF output (C) ABF with MM (a)

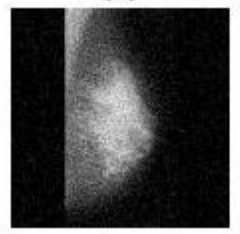

(b)

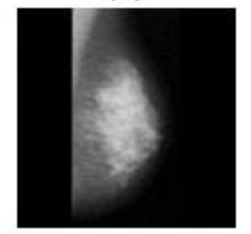

(c)

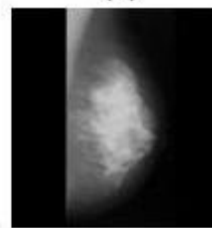

Fig.6 mammogram5: G SPIC B: (a) Input image (b) ABF output (C) ABF with MM

\subsection{Quantitative Evaluation \\ 3.1.1 Mean Square Error}

Averaging the squared intensity of the original (input) image and the resultant (output) image pixels.

$$
M S E=\frac{1}{N M} \sum_{m=0}^{M-1} \sum_{n=0}^{N-1} e^{(m, n)^{2}}
$$

Where $e^{(m, n)}$ is the error difference between the original and the distorted images.

\subsubsection{Peak Signal-to-Noise Ratio (PSNR)}

Peak Signal-to-Noise Ratio (PSNR) and Signal-to-noise ratio (SNR) is a mathematical measure of image quality based on the pixel difference between two images [8] The SNR measure is an estimate of quality of reconstructed image compared with original image. PSNR is defined as :

$$
P S N R=10 \log \frac{s^{2}}{M S E}
$$

Where $\mathrm{s}=255$ for an 8-bit image. The PSNR is basically the SNR when all pixel values are equal to the maximum possible value.

\subsubsection{The structural similarity index:}

SSIM: The structural similarity index is a method for measuring the similarity between two images [9]. The SSIM index is a full reference metric, or we can say the measuring of image quality based on an initial uncompressed or distortion-free image as reference. It compares two images using information about luminous, contrast and structure. SSIM metric is designed to improve on traditional methods like PSNR and MSE and this is calculated on various windows of an image. The measure between two windows $x$ and $\mathrm{y}$ of common size $\mathrm{N} \times \mathrm{N}$ is given as follows:

$$
\operatorname{SSIM}(x, y)=\frac{\left\{\left(2 \mu_{x} \mu_{y}+C_{1}\right)\left(2 \sigma_{x y}+C_{2}\right)\right\}}{\left\{\left(\mu_{x}^{2}+\mu_{y}^{2}+C_{1}\right)\left(\sigma_{x}^{2}+\sigma_{y}^{2}+C_{2}\right)\right\}}
$$

Where $\mu_{x}$ average of is $x, \mu_{y}$ is average of $y, \sigma_{x} \sigma_{y}$ are standard deviation between the original and processed images pixels, respectively. $C_{1}, C_{2}$ are positive constant chosen empirically to avoid the instability of measure. SSIM is a decimal value between $(-1,1)$.

The performance of the Adaptive Bilateral Filter was shown in table 1 . These spatial filters have been judged by the result of MSE, SSIM and PSNR.

Table 1: performance metric for adaptive bilateral filter for noisy Mammogram images (Gaussian noise)

\begin{tabular}{|c|c|c|c|}
\hline Image & MSE*10 $^{-4}$ & PSNR & SSIM \\
\hline Mammogram 1 & 8.52 & 78.82 & 0.64 \\
\hline Mammogram 2 & 5.39 & 80.81 & 0.70 \\
\hline Mammogram 3 & 5.56 & 80.67 & 0.67 \\
\hline Mammogram 4 & 8.47 & 78.84 & 0.63 \\
\hline Mammogram 5 & 3.15 & 83.14 & 0.70 \\
\hline Mean & 6.2180 & 80.4560 & 0.6680 \\
\hline $\begin{array}{c}\text { Standard } \\
\text { deviation }\end{array}$ & 2.2859 & 1.7792 & 0.0327 \\
\hline
\end{tabular}


By applying $\mathrm{ABF}$ on five mammogram images it's found that the mean for MSE, PSNR and SSIM is equal to 6.2180, 80.4560 and 0.6680 respectively and the standard deviation is equal to $2.2859,1.7792$ and 0.0327 respectively.

Table2: performance metric for Morphological adaptive bilateral filter with structure elements- 'disc' dimensions-3 $\mathbf{x} 3$

\begin{tabular}{|c|c|c|c|}
\hline Image & MSE*10^ $^{\wedge}$ - & PSNR & SSIM \\
\hline Mammogram 1 & 6.56 & 80.06 & 0.92 \\
\hline Mammogram 2 & 3.60 & 82.56 & 0.93 \\
\hline Mammogram 3 & 3.64 & 82.50 & 0.93 \\
\hline Mammogram 4 & 6.26 & 80.16 & 0.92 \\
\hline Mammogram 5 & 1.62 & 86.01 & 0.93 \\
\hline Mean & 4.3360 & 82.2580 & 0.9260 \\
\hline $\begin{array}{c}\text { Standard } \\
\text { deviation }\end{array}$ & 2.0646 & 2.4218 & 0.0055 \\
\hline
\end{tabular}

By applying the proposed method using a disk structure elements- 'disc' dimensions-3 x 3. On five mammogram images it's found that the mean for MSE, PSNR and SSIM is equal to $4.3360,82.2580$ and 0.9260 respectively and the standard deviation is equal to $2.0646,2.4218$ and 0.0055 respectively.

Table 3: performance metric for Morphological adaptive bilateral filter with structure elements- 'disc' dimensions-5 $\mathbf{x} 5$

\begin{tabular}{|c|c|c|c|}
\hline Image & MSE*10^- 4 $^{\wedge}$ & PSNR & SSIM \\
\hline Mammogram 1 & 7.16 & 79.58 & 0.88 \\
\hline Mammogram 2 & 3.87 & 82.25 & 0.91 \\
\hline Mammogram 3 & 4.15 & 81.94 & 0.91 \\
\hline Mammogram 4 & 6.58 & 79.94 & 0.92 \\
\hline Mammogram 5 & 1.99 & 85.13 & 0.90 \\
\hline Mean & 4.7500 & 81.7680 & 0.9040 \\
\hline $\begin{array}{c}\text { Standard } \\
\text { deviation }\end{array}$ & 2.1160 & 2.2189 & 0.0152 \\
\hline
\end{tabular}

By applying the proposed method using a disk structure elements- 'disc' dimensions - 5x5. On five mammogram images it's found that the mean for, PSNR and SSIM is equal to $4.7500, \quad 81.7680$ and 0.9040 respectively and the standard deviation is equal to $2.1160,2.2189$ and 0.0152 respectively.
Table 4: performance metric for Morphological adaptive bilateral filter with structure elements- 'disc' dimensions-7 $\mathbf{x} 7$

\begin{tabular}{|c|c|c|c|}
\hline Image & MSE $^{*} \mathbf{1 0}^{\wedge} \mathbf{- 4}$ & PSNR & SSIM \\
\hline Mammogram 1 & 12 & 77.14 & 0.86 \\
\hline Mammogram 2 & 5.23 & 80.94 & 0.88 \\
\hline Mammogram 3 & 5.40 & 80.79 & 0.87 \\
\hline Mammogram 4 & 7.25 & 79.36 & 0.84 \\
\hline Mammogram 5 & 2.72 & 83.78 & 0.82 \\
\hline Mean & 6.5200 & 80.4020 & 0.8540 \\
\hline $\begin{array}{c}\text { Standard } \\
\text { deviation }\end{array}$ & 3.4613 & 2.4278 & 0.0241 \\
\hline
\end{tabular}

By applying the proposed method using a disk structure elements- 'disc' dimensions-7 x 7. On five mammogram images it's found that the mean for, PSNR and SSIM is equal to $6.5200,80.4020$ and 0.8540 respectively and the standard deviation is equal to $3.4613,2.4278$ and 0.0241 respectively.

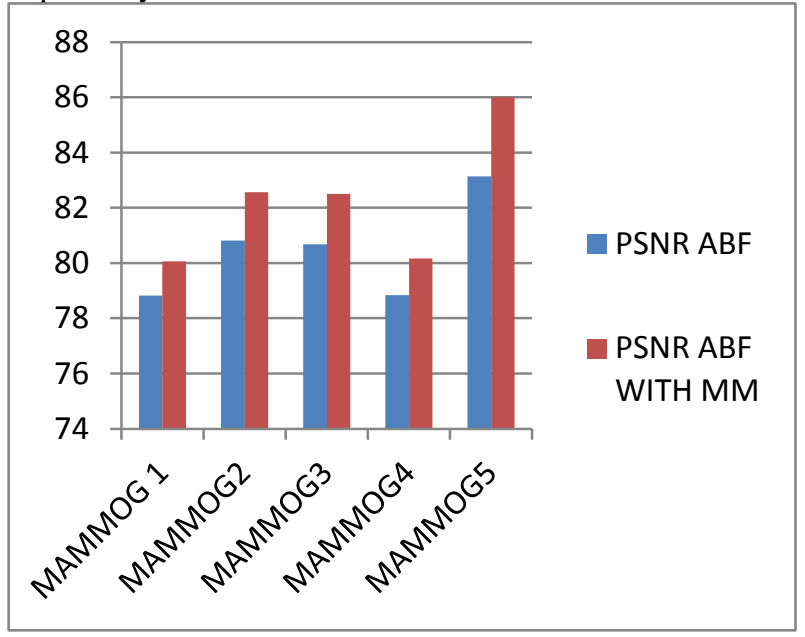

Fig 7: A comparison of ABF and ABF with MM w.r.t. PSNR

From the above figure it's observed that the proposed method produced a noise removal image signal with noticeably improved perceptual quality compared to the original bilateral filtering method. 


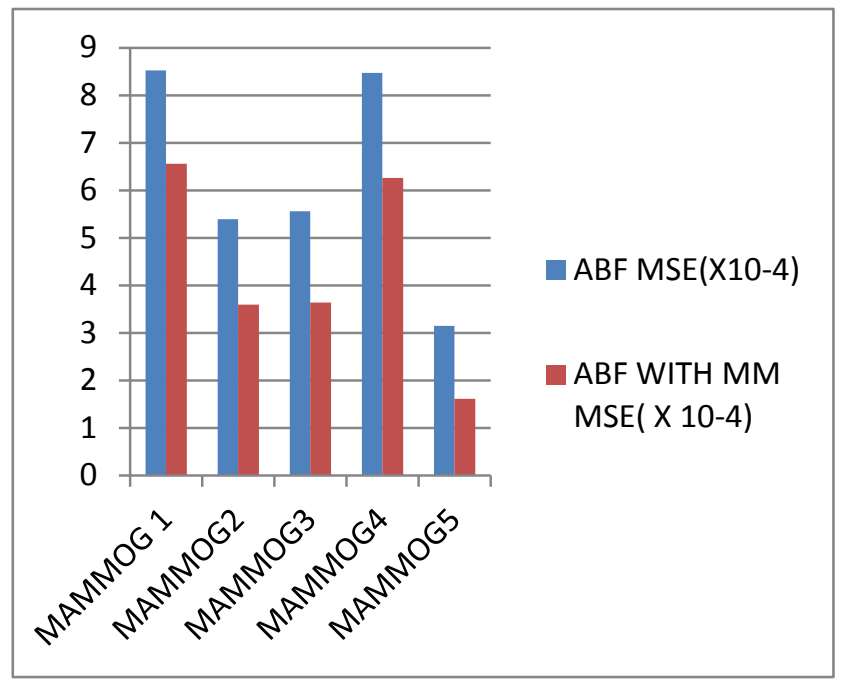

Fig 8: A comparison of $\mathrm{ABF}$ and $\mathrm{ABF}$ with $\mathrm{MM}$ w.r.t. MSE

As Shown in fig: 8 The MSE value is reduced using MM operators with combination of ABF.

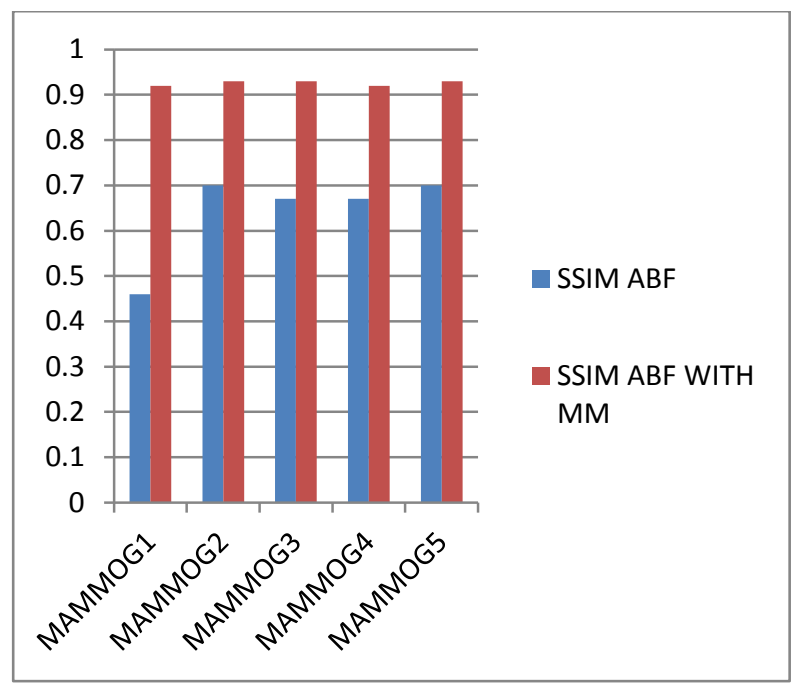

Fig 9: A comparison of $\mathrm{ABF}$ and $\mathrm{ABF}$ with $\mathrm{MM}$ w.r.t. SSIM

Figure 9: shows that using structure similarity index (SSIM) as a parameter it's found that morphological operators produce less change in the image structure than using adaptive bilateral filter alone, so the result image is significantly more closer to the reference image.

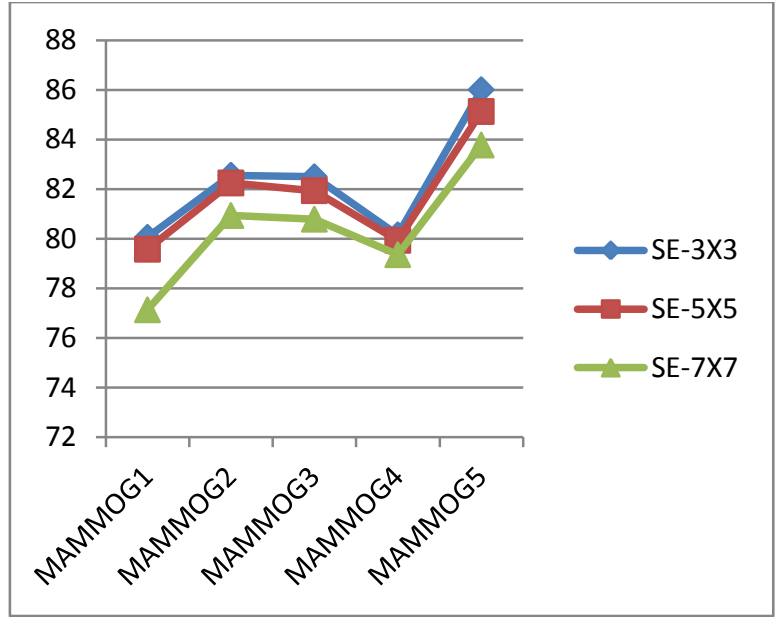

Fig 10: PSNR VS SE dimension

Figure 10: shows the estimated PSNR versus a structure element dimension. It is observed that the lowest dimension of SE i.e. $3 \times 3$ has given the high PSNR value with given window width of the filter.

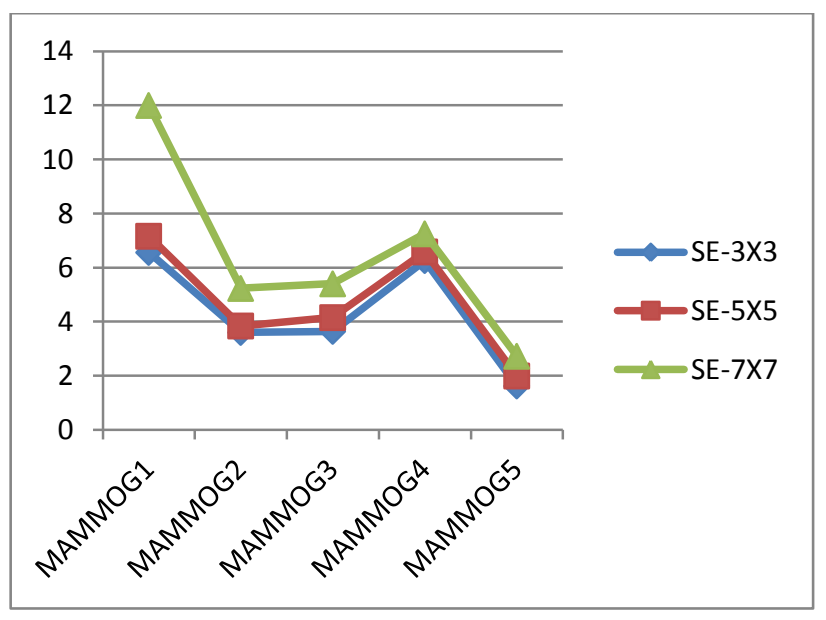

Fig 11: MSE VS SE dimension

Figure 11: shows the MSE versus structure element dimensions. It is observed that the lowest dimension of SE i.e. 3 X 3 has given the minimum MSE value with a given window width of the filter.

From the above results it's obvious that the morphological operators improve the performance of adaptive bilateral filter and overall appearance of the output images, it has found that combination of those techniques helps to restore the original image up to $92 \%$ obtained from structure similarity index (SSIM), with 82 peak signal to noise ratio and very small mean square error $4.33 * 10-4$ obtained as average results from 5 mammogram images.

It was observed that if the width of window exceed the range [4 7] or structure element chosen is other than a disc or is exceeding the dimensions more than the $7 \times 7$ the output image is getting blurred, there for many techniques to debluring the image (e.g. image restoration via debluring or what so called deconvolution ) or sharpen it back was used ( e.g. image unsharpen masking and sharpening by laplacian operators), but none of them had a great impact on a visual quality of the image and some even had dropped the PSNR and SSIM. 


\section{CONCLUSION}

This work has presented adaptive bilateral filters (ABF) combined with morphological operators, to enhance the quality of the noise distorted mammographic images. From the previous results, it has been proved that morphological operation increases the efficiency of the $\mathrm{ABF}$ and the quality appearance of the output images, which was experimented on various types of mammogram images. Design parameters are evaluated using quality metrics namely mean square error (MSE), peak signal to noise ratio (PSNR) and structure similarity index (SSIM). It was observed that as the size of the structure element increase, the output image is getting blurred.

The optimization procedure which was developed to optimize $\mathrm{ABF}$ parameters automatically had found that the combination of those techniques helps to restore the original image up to 92\% obtained from structure similarity index (SSIM), with 82 peak signal to noise ratio and very small mean square error $4.33 * 10^{-4}$ obtained as average results from 5 mammogram images.

For the future work, it is recommended that this method is to be generalized to other types of noise like Poisson, speckle and salt and pepper noise. Also developed an algorithm that allows using an adaptive structure element is recommended.

\section{REFERENCES}

[1] Bozek, J., Delac, K., \& Grgic, M. (2008, September). Computer-aided detection and diagnosis of breast abnormalities in digital mammography. In ELMAR, 2008. 50th International Symposium (Vol. 1, pp. 45-52). IEEE.
[2] Verma, R., \& Ali, D. J. (2013). A comparative study of various types of image noise and efficient noise removal techniques. International journal of advanced research in computer science and software engineering, 3(10).

[3] Zhang, B., \& Allebach, J. P. (2008). Adaptive bilateral filter for sharpness enhancement and noise removal IEEE transactions on Image Processing, 17(5), 664-678.

[4] Reinhardt, J. M., \& Higgins, W. E. (1996). Efficient morphological shape representation. IEEE Transactions on Image Processing, 5(1), 89-101.

[5] Gonzalez, R. C., Woods, R. E., \& Eddins, S. L. (2004). Digital Imaging Processing Using MATLAB.

[6] Tomasi, C., \& Manduchi, R. (1998, January). Bilateral filtering for gray and color images. In Computer Vision, 1998. Sixth International Conference on (pp. 839-846). IEEE.

[7] Salembier, P., \& Serra, J. (1995). Flat zones filtering, connected operators, and filters by reconstruction. IEEE Transactions on image processing, 4(8), 1153-1160.

[8] Banerjee, I., Bhattacharyya, S., \& Sanyal, G. (2013). Study and analysis of steganography with pixel factor mapping (PFM) method. International Journal of Application or Innovation in Engineering \& Management (IJAIEM), 2(8), 258-266.

[9] Sampat, M. P., Wang, Z., Gupta, S., Bovik, A. C., \& Markey, M. K. (2009). Complex wavelet structural similarity: A new image similarity index. IEEE transactions on image processing, 18(11), 2385-2401. 